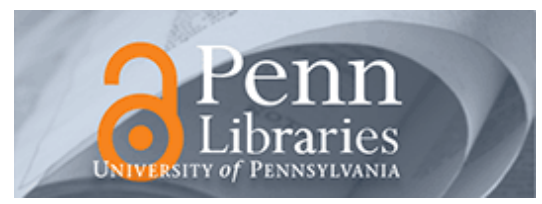

University of Pennsylvania

ScholarlyCommons

\title{
A Data-Driven Appearance Model for Human Fatigue
}

Joseph T. Kider Jr.

University of Pennsylvania, kiderj@seas.upenn.edu

Kaitlin Pollock

University of Pennsylvania

Alla Safonova

University of Pennsylvania, alla@cis.upenn.edu

Follow this and additional works at: https://repository.upenn.edu/cis_papers

Part of the Computer Sciences Commons

\section{Recommended Citation}

Joseph T. Kider Jr., Kaitlin Pollock, and Alla Safonova, "A Data-Driven Appearance Model for Human Fatigue", . January 2011.

Kider, J., Pollock, K., \& Safonova, A., A Data-Driven Appearance Model for Human Fatigue, Eurographics/ACM SIGGRAPH Symposium on Computer Animation, SCA 2011, doi: 10.2312/SCA

ACM COPYRIGHT NOTICE. Copyright (C 2011 by the Association for Computing Machinery, Inc. Permission to make digital or hard copies of part or all of this work for personal or classroom use is granted without fee provided that copies are not made or distributed for profit or commercial advantage and that copies bear this notice and the full citation on the first page. Copyrights for components of this work owned by others than ACM must be honored. Abstracting with credit is permitted. To copy otherwise, to republish, to post on servers, or to redistribute to lists, requires prior specific permission and/or a fee. Request permissions from Publications Dept., ACM, Inc., fax +1 (212) 869-0481, or permissions@acm.org.

This paper is posted at ScholarlyCommons. https://repository.upenn.edu/cis_papers/670

For more information, please contact repository@pobox.upenn.edu. 


\title{
A Data-Driven Appearance Model for Human Fatigue
}

\begin{abstract}
Humans become visibly tired during physical activity. After a set of squats, jumping jacks or walking up a flight of stairs, individuals start to pant, sweat, loose their balance, and flush. Simulating these physiological changes due to exertion and exhaustion on an animated character greatly enhances a motion's realism. These fatigue factors depend on the mechanical, physical, and biochemical function states of the human body. The difficulty of simulating fatigue for character animation is due in part to the complex anatomy of the human body. We present a multi-modal capturing technique for acquiring synchronized biosignal data and motion capture data to enhance character animation. The fatigue model utilizes an anatomically derived model of the human body that includes a torso, organs, face, and rigged body. This model is then driven by biosignal output. Our animations show the wide range of exhaustion behaviors synthesized from real biological data output. We demonstrate the fatigue model by augmenting standard motion capture with exhaustion effects to produce more realistic appearance changes during three exercise examples. We compare the fatigue model with both simple procedural methods and a dense marker set data capture of exercise motions.

Disciplines

Computer Sciences

\section{Comments}

Kider, J., Pollock, K., \& Safonova, A., A Data-Driven Appearance Model for Human Fatigue, Eurographics/ ACM SIGGRAPH Symposium on Computer Animation, SCA 2011, doi: 10.2312/SCA

ACM COPYRIGHT NOTICE. Copyright @ 2011 by the Association for Computing Machinery, Inc.

Permission to make digital or hard copies of part or all of this work for personal or classroom use is granted without fee provided that copies are not made or distributed for profit or commercial advantage and that copies bear this notice and the full citation on the first page. Copyrights for components of this work owned by others than ACM must be honored. Abstracting with credit is permitted. To copy otherwise, to republish, to post on servers, or to redistribute to lists, requires prior specific permission and/or a fee. Request permissions from Publications Dept., ACM, Inc., fax +1 (212) 869-0481, or permissions@acm.org.
\end{abstract}




\title{
A Data-driven Appearance Model for Human Fatigue
}

\author{
Joseph T. Kider Jr., Kaitlin Pollock, and Alla Safonova
}

University of Pennsylvania

\begin{abstract}
Humans become visibly tired during physical activity. After a set of squats, jumping jacks or walking up a fight of stairs, individuals start to pant, sweat, loose their balance, and flush. Simulating these physiological changes due to exertion and exhaustion on an animated character greatly enhances a motion's realism. These fatigue factors depend on the mechanical, physical, and biochemical function states of the human body. The difficulty of simulating fatigue for character animation is due in part to the complex anatomy of the human body. We present a multi-modal capturing technique for acquiring synchronized biosignal data and motion capture data to enhance character animation. The fatigue model utilizes an anatomically derived model of the human body that includes a torso, organs, face, and rigged body. This model is then driven by biosignal output. Our animations show the wide range of exhaustion behaviors synthesized from real biological data output. We demonstrate the fatigue model by augmenting standard motion capture with exhaustion effects to produce more realistic appearance changes during three exercise examples. We compare the fatigue model with both simple procedural methods and a dense marker set data capture of exercise motions.
\end{abstract}

Categories and Subject Descriptors (according to ACM CCS): I.3.3 [Computer Graphics]: Three-Dimensional Graphics and Realism-Computer Animation

\section{Introduction}

Currently in animated films and video games, the visualization of human exhaustion is either artistically stylized or ignored completely. Every human sweats, pants, flushes, and loses balance as they perform physical activity (Figure 1). No one has the fitness level of an animated character. Running up a hill or climbing stairs has a physiological effect on the body. Therefore the physiological effects of fatigue cannot be ignored when producing realistic animations. Traditionally, artists tediously paint textures and meticulously layer subtle motions to produce dynamic exhaustion effects. This process is ad hoc, tedious and time-consuming for animators to tune the visual effect. We present a novel datadriven approach to automatically simulate exhaustion effects on virtual characters so they appear more tired the longer they perform motions. This work enhances animation by taking advantage of biomedical research and exercise physiology to apply captured data on anatomically inspired model.

Traditional motion capture techniques produce believable character animation. However, traditional 30-60 data marker sets only approximate change in human motion. As virtual characters become more realistic, it is important to simulate physiological appearance changes in addition to motion capture to simulate the body's response to physical activity. Exhaustion effects are particularly challenging since they are non-periodic and complex, involving both motions and appearance changes.

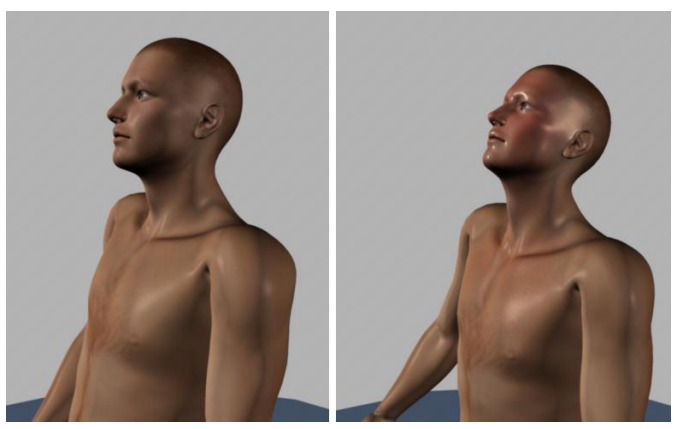

Figure 1: This figure shows the first frame and the last frame of a jumping jack exercise. On the right, the character appears red, sweaty, and moves his head back since he feels fatigued from jumping for 2 minutes.

In this paper, we introduce a data-driven method to capture and animate physiologically apparent exhaustion ef- 
fects on a human character. We propose a multi-modal motion capture approach for capturing both human motion and biosignal data (EKG, BVP, GSR, respiration, and skin temperature) visualized in Figure 2. This novel capture process greatly enhances the quality of the data we collect. Our objective is to apply this data onto a human character to enhance secondary exhaustion animation effects such as sweating, breathing, and flushing (Figure 1). We accurately recreate an anatomically inspired human body to demonstrate the exhaustion effects. The human model consists of a rigged skeleton, lungs, ribs, spine, intestines, heart, and face. The biosignal data controls the deformation of the human model. This allows us to automatically generate exhaustion effects layered on human motion. We propose a straightforward method for producing a data-driven appearance model of human fatigue.

To validate the fatigue model, we captured exercise motions of ten subjects (five males and five females) ranging in size and shape. The subjects all exhibited the same significant signs of exhaustion when they performed the exercise motions. We illustrate these physiological changes of exercise by showing biosignal output plots. Physical activity produces significant change in the plots of respiration rate, pulse, skin temperature, and galvanic skin response. We demonstrate the fatigue output on a virtual character whose exhaustion effects change due to the biosignal output signals for three types of exercise motions: jumping jacks, stair stepping, and squatting. Lastly, we evaluate the results by comparing the simulated model to a dense motion capture marker set that covers the chest. The fatigue model produces similar chest deformations without needing to capture $50 \mathrm{ex}$ tra chest markers. Additionally, the fatigue model is able to add flushing and sweating to the virtual characters, which cannot be captured effectively with previous methods.

The main contribution of this work is presenting a biosignal data capture technique that drives a human character to augment its visual appearance. We layer various dynamic physiological effects such as perspiration, flushing, and breathing on the human character. These effects change due to the biodata output providing more realistic appearance of fatigue on the character animation.

\section{Related Work}

To our knowledge, little work has been conducted to enhance virtual character animations with synchronized biodata output. In the movie Beowulf, Sony Picture Imageworks combined electrooculography (EOG) with motion capture to create realistic eye movement on characters. Some researchers [SCCH09, TLX10, MTCS08] capture electromyography (EMG) with motion capture to measure and record the electrical activity produced by skeletal muscles. Unfortunately, full body EMG systems are extremely expensive, very invasive for the subject, and produce extremely noisy data. These biodata enhancements are cheaper, non-invasive,

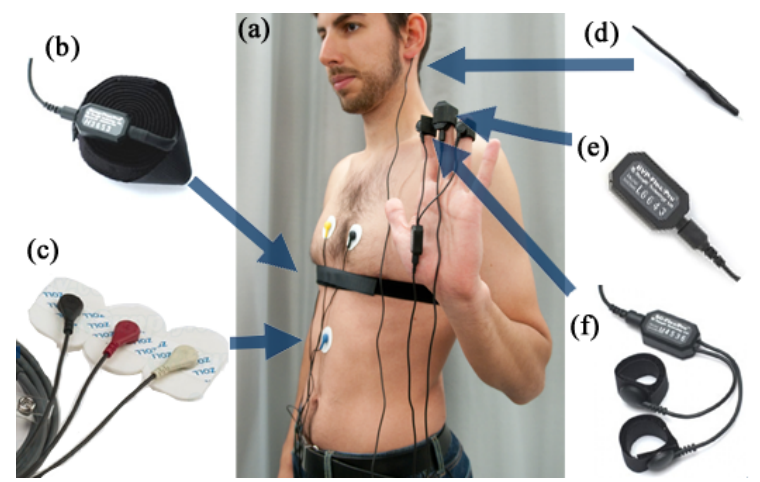

Figure 2: This figure shows the biodata sensor enhancements to the motion capture process. The subject (a) is asked to wear five different sensors: (b) a respiration sensor that wraps around the chest, (c) an electrocardiography sensor with three leads on the chest, $(d)$ external skin temperature sensor behind the ear, (e) blood volume pulse sensor and (f) galvanic skin response sensor on the left hand. These sensors are minimally invasive and only take an additionally five minutes to place on the subject.

and produce cleaner data which can be directly applied to enhance animations.

The simulation and visual appearance of anatomical secondary animation effects is well studied in graphics. Monheit and Badler [MB91] simulated a flexible torso and spine. Lee and Terzopoulos [LT06] introduced an animated biomechanical model for the human neck.. Promayon et al. [PBP97] looked at the rib cage, lungs, diaphragm, and abdomen during calm breathing patterns. Kaye et al. [KMP97] modeled a basic cardiorespiratory physiology of the body based on CT scans.

Zordan et al. [ZCCD06] presented an anatomically inspired model of the human torso to simulate realistic respiration on human characters. Their torso simulation reconstructed the human rib cage, spine, lungs, diaphragm muscle, and lower abdomen. Characters became more alive with simulated breathing motions. Sanders et al. [SDZB08] improved the technique by placing 40 additional markers on the human torso as input for the system. We compare the $f a$ tigue model's results to a similar dense marker set found in this paper. DiLorenzo et al. [Di109, DZS08] presented a technique for using sound tracks to drive the breathing model for laughing, coughing, and sneezing. The underlying model of the rib cage, spine and lungs follow a similar model to this approach. We use output from the biosensors to drive the fatigue model, rather than a dense marker set. Additionally, our approach simulates heartbeats, flushing, mouth motions and perspiration.

Many researchers have simulated facial movements [PB81,KHYS02,Wat87,SNF05]. Sagar [Sag06] and Bradley 
et al. [BHPS10] captured facial expressions, geometry, and textures to apply to facial animations. The fatigue model does not simulate facial muscles or utilize an expensive performance capture technique; instead it uses data-derived blend shapes for the facial motion from sample facial captures. This motion is guided by the respiration data to modify the blend shape of the underlying facial rigs [DCFN06] to simulate mouth breathing.

The appearance of human skin, specifically for the face and neck, contribute to showing the level of fatigue. Igarashi et al. [INN07] survey various appearance models for skin. Kalra and Magnenat-Thalmann [KMT02] first proposed modeling facial color changes based on a texturing approach. Weyrich et al. [WMP* 06] looked at skin measurements under various hot and sweaty conditions. Yamada and Watanabe [YW07] observed facial color change during anger. Melo and Gratch [MG09] propose a method to layer sweating and blushing on virtual characters. They focused on interactions with other characters so the blushing and sweating were caused by emotional stress and not thermoregulation.

Jimenez et al. [JSB*10] captured real subject measurements of melanin and hemoglobin concentrations to dynamically model the appearance of facial color. For one of the subject tests, a person ran stairs and measurements were taken once a minute. The facial color appearance model is based on this approach for simulating the skin color changes during the exercise motions. However, we use real biosignal data to drive the appearance rig parameters.

Methods have been proposed to capture and animate the complex skin deformations of the human body. Allen et al. [ACP02] calculated skeleton-driven body deformations by utilizing range scan data. Sand et. al. [SMP03] presented a method to acquire deformations based on silhouettes. Park and Hodgins [PH06] captured subtle movements of skin during dynamic movements with a high dimensional (350) marker set, with 50 of these on the chest alone. They produced very realistic effects for breathing, jiggling, and bulging. However, the marker setup is not only uncomfortable to wear, but the data is tedious to clean and label. Their follow up work [PH08] utilized the capture database of skin deformations to produce a statistical model to layer skin deformations on various motions.

The fatigue model differs in important ways from these skin performance capture techniques. Foremost, the fatigue model's data capture is less intrusive to the subject, and takes less time to clean and process the motion data. Though skin performance techniques produce highly realistic results for subtle deformations, we are primarily concerned with breathing in this paper. The fatigue model simulates the underlying anatomical model from output of the biodata to simulate realistic chest movement. Additionally, we simulate a variety of fatigue effects such as sweating and flushing which could not be captured by these techniques alone.

\section{Exercise Mechanics}

As humans perform physical activity they put their body under stress. The more intense the motion is the more intense the body's physiological reaction. Exercise presents one of the highest levels of stress on the human body. Major signs of fatigue include: changes in respiration, heart rate, perspiration, temperature changes, and flushing. The system attempts to model these major changes so the virtual characters appear more fatigued as they perform motions. There are two types of motion classes we are looking at: anaerobic (squats) where the muscles are exerted for a short period of time, and aerobic (jumping jacks, stair stepping) performed for a longer period of time.

As humans begin physical activity, their respiration mechanics change. Breathing becomes deeper and more vigorous. This breathing pattern is called hyperpnea. The respiratory changes seen in hyperpnea match metabolic demands of the body. First, ventilation increases abruptly as exercise begins. The excitatory impulses from proprioceptors in the muscles and tendons activate the respiratory centers. Ventilation then declines suddenly after exercise stops [Mar98, Min93, Wes04].

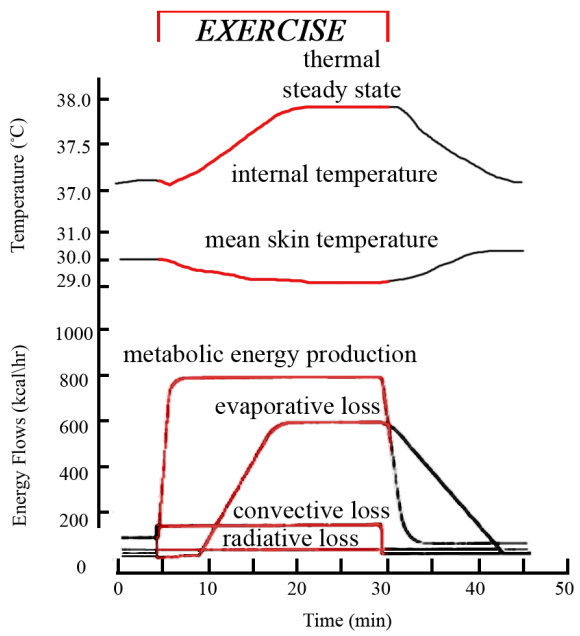

Figure 4: These graphs show the changes in the heat flow rates and body temperature during exercise.

To prolong motions for extended periods of time, the heart must simultaneously supply enough blood flow to muscles, ensure oxygen is distributed in the body, and move adequate blood to the skin so heat can dissipate to meet the body's thermoregulatory demands [LM88]. As the heart pumps more blood to meet the demands of oxygen in the body tissues, it recruits more muscle fibers. This increases the force required to perform the blood flow movement. The heart's contraction and expansion increases. The blood rushing to the body's tissues changes both the internal and external temperature. Warm blood returns to the body's core. 


\section{Our Model's Pipeline}
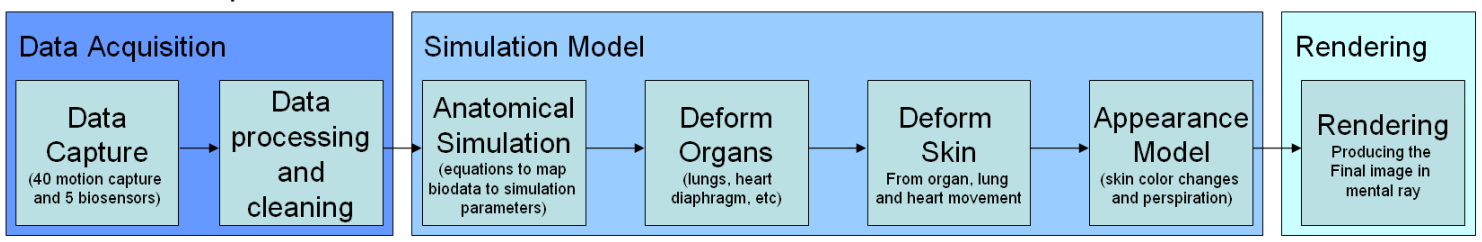

Figure 3: This figure shows an overview of the simulation pipeline. We capture the data, clean the motion capture take, apply the biodata to the simulation parameters, deform the organs, which in turn deform the skin, change the appearance of the figure (sweating, flushing), then render the final output.

The muscles contract causing vasoconstriction to reduce the blood flow. This makes peripheral skin temperature decrease due to the sympathetic nervous system during intense motions. This thermoregulation change can be seen in Figure 4 [LM88].

The facial skin tissue has more capillary loops per area then other parts of the body. Vasodilation (widening of blood vessels) occurs in the face producing a blushing effect [MAP*82]. Jimenez et al. [JSB*10] related this effect to a "texture look up" of hemoglobin and melanin values.

The sympathetic nervous system directly controls sweat gland activity and skin pore size. Skin conductance measures how skin can conduct current. This galvanic skin response is directly proportional to sweat being produced [PT57].

There are many other factors that contribute to fatigue, such as lactic acid build up, hydrogen ion accumulation and glycogen depletion. Though these factors are important in human fatigue, we do not consider them in the paper since they require blood work, and other invasive measuring methods to properly measure.

\section{System Overview}

We introduce a data-driven method to capture and animate fatigue effects on a human character. An overview of the $f a$ tigue model is found in Figure 3. First, we capture the motion capture data on the subject. We use a standard 40 motion capture marker setup, augmented with 5 biosignal sensors. We then clean and process the data. The next stage simulates the underlying anatomically derived model. We use the output from the biosignal sensors to drive the anatomical simulation which relates the data into parameters which can be used to deform the organs. We use these parameters to drive the underlying organs: heart, lungs, etc. The organ simulation deforms the skin of the virtual character so it appears alive. We then adjust the character's outer appearance from the biodata parameters making the character flush and sweat as the data changes. Finally, we render the final output in the scene.

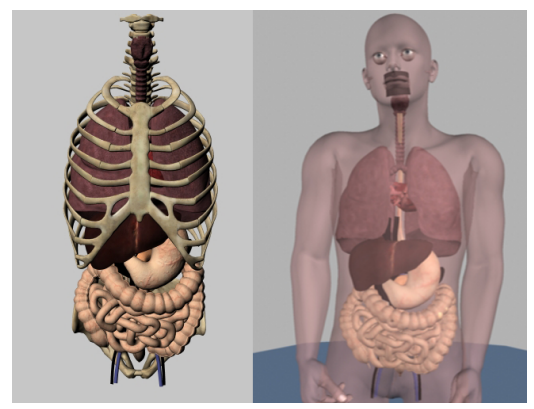

Figure 5: The left image shows the internal anatomy of the virtual human. The right image shows an x-ray view of the organs inside the body.

\section{Data Acquisition}

For an experimental analysis we captured 10 subjects (5 males and 5 females) of various weights (48kg to $108 \mathrm{~kg}$ ) and sizes $(157 \mathrm{~cm}$ to $195 \mathrm{~cm})$. The subjects ranged between 20 to 34 years of age and had a variety of fitness levels according to their responses on a questionnaire. Each subject performed at least 2 exercise types: jumping jacks, squats, or stair aerobics giving us around 25 unique exercise sample motions. We have made the data publicly available at: http://cg.cis.upenn.edu/hms/research/Exhaustion/.

\subsection{Motion Capture}

We used a commercial 16 near infra-red camera optical motion capture system from Vicon [vic11]. The motion data was captured at 120 frames per second in a $3 \mathrm{~m}$ by $3 \mathrm{~m}$ by $3 \mathrm{~m}$ capture volume. We used a standard 40 retro-reflective marker setup for the motions. Additionally, we placed approximately 50 markers on the chest and 16 markers on the face so we could compare deformations, capturing, and cleaning times. We captured, reconstructed, and cleaned the marker data using VICON BLADE 1.6 [vic11]. The motion was then transferred to a skinned virtual character in Maya. 


\subsection{Biosignal Sensors}

We captured five biosignal sensors in addition to the motion capture data. These sensors are illustrated in Figure 2. We used a ProComp Infiniti encoder to wirelessly capture the data on a subject at a rate of 2040 samples per second. To synchronize the sensors we used a 5 volt TTL signal generated when a motion capture session started. This signal was captured as a sixth channel in the encoder and used to synchronize the motion after the capture process.

Respiration: The respiration sensor is placed around the subject's upper chest. The sensor measures the abdominal expansion of a subject during respiration. The device stretches and contracts as the subject breaths and records the signal's waveform.

Electrocardiography (EKG): An EKG signal detects and amplifies the small electrical voltage the heart muscle fibers generates when it contracts and expands. The EKG sensor used 3 leads: a negative sensor on the right shoulder, the positive sensor on the lower center of the chest, and the ground sensor on the right shoulder area.

Peripheral skin temperature: This sensor is a 0.125 inch thermistor that detects changes in skin temperature. The skin temperature changes as blood perfuses to the skin. We placed the sensor on the back of the ear to measure the skin temperature of the upper body.

Blood Volume Pulse (BVP): bounces infra-red light against the skin and measures the reflected light; this sensor is also commonly referred to as photoplethysmography in the literature. We attached this sensor to the middle finger.

Galvanic Skin Response (GSR): This sensor measures the skin conductance of electricity. A small voltage is applied between the two sensors placed on the index and fourth finger. Skin conductance correlates with the sweat gland and skin pore size of the subject.

We filter the EKG signal after capturing. It is measured in microvolts. This output gives us a waveform signal that we further process. First, we run a low frequency filter set at either $0.5 \mathrm{~Hz}$ or $1 \mathrm{~Hz}$ and high frequency filter set at $40 \mathrm{~Hz}$. This limits artifacts for routine cardiac rhythm monitoring. The high-pass filter helps reduce artifacts and the low-pass filter helps reduce $60 \mathrm{~Hz}$ power line noise.

\section{Simulation Model}

The virtual human characters have an anatomical model of the internal organs, such as the lungs, heart, esophagus, and lower trunk [Mar98]. We have also added a rib cage and spine [MB91, SDZB08]. This internal anatomy moves and deforms based on biosignal data output. We visualize our internal model and an x-ray view in our virtual character in Figure 5. We derive equations inspired from the exercise physiology literature [PT57, MAP*82, LM88, Wes04,
Min93]. These equations map the biosignal sensor output to our organ deformations and visual appearance changes.

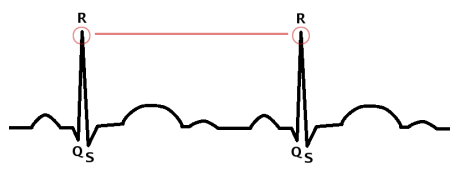

Figure 6: The QRS complex deflections output from the EKG signal. We measure the distance between the $R$ peaks and their amplitude to schedule the virtual heartbeat.

Heart Deformation: The EKG signal drives the deformation of the human model's heart. The signal usually forms a QRS complex interval (Figure 6) for the peaks and valleys of a heart beat. The interval between two $\mathrm{R}$ waves is the inverse of the heart rate. The peaks can be easily found with the findpeaks() function in matlab. We calculate the interbeat interval (IBI) and heart rate (HR) as follows to pulse the human model's heart:

$$
\begin{gathered}
I B I=\frac{1500}{(\text { distance between R peaks in } \mathrm{mm})} \\
H R=\frac{60000}{I B I}
\end{gathered}
$$

These equations are derived from human physiology and provide heartbeat intervals that are used to deform the heart shape. The values 1500 and 60,000 are constants to read the EKG signal information [Mar98]. The volume of the heart is roughly approximated $240 \mathrm{~mL}$ (average in healthy adults). The stroke volume $(S V)$ for a healthy adult is $70 \mathrm{~mL}$. This is the blood pumped during each beat. We scale this based on the amplitude of the QRS complex. So as the signal rises, the heart pumps more blood and deforms more in the chest. This result is very subtle.

Flushing effect: The BVP and temperature sensors change the human visual appearance to flush and sweat. Changes in the BVP sensor represent changes in the blood volume throughout the body. The signal has two components: pulse and amplitude. As the amplitude of the BVP deviation increases this correlates to an increase in vasoconstriction of arterioles in the viscera and skin, which simply means blood flow greatly increases. As this occurs, flushing levels rise.

Similar to Jimenez et al. [JSB*10], before the data capture, we derived an appearance rig shader. We added the upper chest, however, as it also tends to change color. We captured sample photos of one male's and one female's face and chest and constructed a data derived appearance rig. This gives a texture we can index into as the biosignal channels change. Therefore, we approximate the increase in flushing by linearly scaling based on flushing = $1 /$ temperature $+B V P_{a m p}$. We derived this metric from the underlying anatomy [MAP*82, Mar98]; which gives us a 
perceptual metric to make the human characters flush as they exhaust.

Sweating model: Perspiration is modeled from the GSR biosignal. We layer sweat particles and change the specularity of the skin's subsurface scattering layers to simulate sweating. The relationship between active sweat glands and skin conductance is linear [PT57]. Here $G_{t}$ is the total conductance we measure from the sensor, $\mathrm{N}$ is the number of unknown sweat glands that are currently active. $G_{g}$ we represent as a constant, which represents the average conductance increment of each active gland. $G_{a}$ is a constant. This is the average value for an additional conducting path whose contribution to the total conductance is not related to the the test area. We set these values to 0.18 and 0.08 respectively. These constants were derived from the empirical experiments of Price et al. [PT57]. The equations to solve for the active sweat glands are as follows.

$$
\begin{gathered}
G_{t}=G_{a}+G_{g} N \\
N=\frac{G_{t}-G_{a}}{G_{g}}
\end{gathered}
$$

Lung Deformation: The respiration output drives the lung deformations. We simplified the lung model so that we modeled both lungs as only having a single compartment [Met97]. The respiration sensor measures the relative amount of expansion. The average lung volume is 6 liters in men, and 4.2 liters in women. The total lung capacity $T L C$ is commonly calculated as the vital capacity $(V C)+$ residual volume $(R V)$. The vital capacity $(V C)$ is calculated from inspiratory reserve volume $(I R V)+$ expiratory reserve volume $(E R V)+$ the tidal volume $\left(V_{t}\right)$. Contracting the diaphragm pulls the bottom of the lung cavity down, increasing the volume and decreasing the pressure. The sensor directly maps the change in the volume of the lungs. We get the starting lung volume measurements $\left(V C, E R V\right.$ and $\left.V_{t}\right)$ from spirometer readings taken from every subject.

Face Deformation: The respiration output also drives the face deformations. Using 16 facial markers, we initially captured datasets from one male and one female face. From this motion we derived blend shapes of mouth and nostril motions for breathing. The system uses the respiration output to determine when a breath occurs and its amplitude. We use these two parameters to drive the facial blend shapes so the character appears to breathe. The subjects therefore do not have to wear facial markers for their capture.

\section{Experimental Results}

In the submission video that accompanies this paper, results are included for three different subjects performing three different motions: jumping jacks, stair stepping, and squats. The subjects show signs of exhaustion as they perform the motion. For rendering, the shaders are developed in Mental Ray and modeled the figures in Autodesk's Maya. The characters are scaled to be proportional to the capture subject's dimensions. The appearance model and character rig can easily fit into an animation pipeline, and only adds a small overhead to apply our exhaustion plug-in simulation and shaders.

\subsection{Analysis of Biodata}

25 different motions were captured from 10 subjects. For every experiment we asked the subjects to idle before the motion, perform the exercise, then idle to cool down afterwards. Figure 11(a) shows the output from all the biosensors (respiration, galvanic skin response, electrocardiography, skin temperature, and blood volume pulse) for one of the subjects performing a jumping jacks exercise. On each graph, the first red line indicates the start of the exercise and the second red line indicates the end of the exercise. As can be readily noted in this figure, there is a major difference in the biodata during an exercise: after the first red line temperature starts to dip, the respiration pattern dramatically changes, GSR rises, and the heart rate and BVP intensify.

Figures 11(b) shows the output from the temperature sensor for four different subjects. The general form of all four curves is the same for all the subjects - after the first red line the temperature starts to dip and it starts going back to normal after the second red line. Figures 11(c) show the output from the respiration sensor for four different subjects. Again, the general form of all four curves is the same across all subjects. This result shows that the exercise experiments are very repeatable across subjects and exercises. Due to space limitation we could not include data for other sensors and for all 10 subject, but the same conclusion holds for the rest of the data.

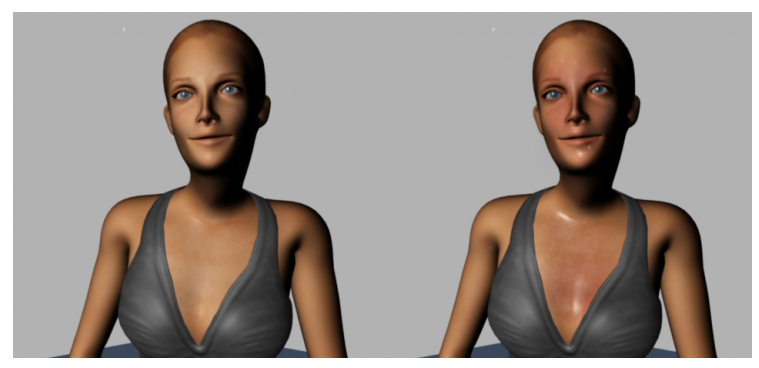

Figure 8: The left image shows the model without any exhaustion effects. The right image shows the model flushing, and perspiring after an exhausting squat exercise.

\subsection{Comparison Results}

We compare chest deformations for the fatigue model with a chest deformation computed from a dense marker set (see Figure 9 for marker setup we used). We capture 40 markers for the body, augmented with 50 markers for the chest, and 


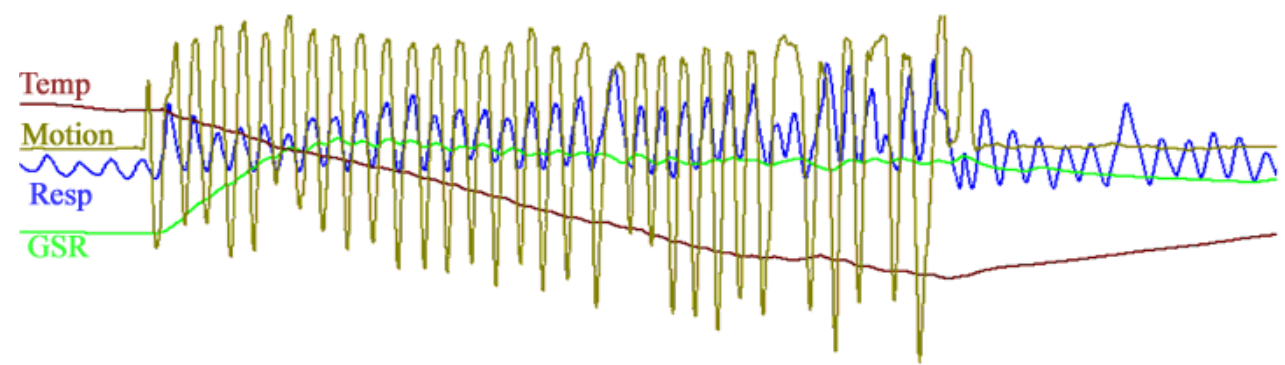

Figure 7: This graph shows an overlay of motion capture and biodata. (Yellow) displays the motion trajectory of the vertical component of the head marker as an unathletic female subject performs her squats. (Blue) corresponds to respiration, (red) skin temperature, and (green) skin conductance. Changes in biodata correlate to changes in motion capture trajectories. In the beginning the breaths are synced with the motion, towards the end of the exercise fatigue sets in: the respiration becomes more chaotic, temperature has changed significantly, and the subject's motion becomes more erratic and starts to decay.

16 markers for the face. The dense marker set directly captures skin deformations and therefore can serve as groundtruth. The fatigue model drives chest deformations indirectly (by deforming underlying organs). Figure 10 is a side-byside comparison of the two methods. Please refer to the submission video for a better comparison that includes a full animation. There is no noticeable difference between the two models. Therefore, the fatigue model achieves similar chest deformations while being an order of magnitude less intrusive and invasive than the dense marker set method.

The submission video also compares a simple sine wave output to deform the chest. This method drives the lungs and heart, and makes characters feel more alive. However, looking at Figure 11 we see that respiration and heart rate signals change dramatically over time and are non-uniform. Therefore, the fatigue model realistically follows how humans breathe, flush, and sweat as they perform different physical activities. For example, the respiration changes dramatically between idling and performing an intensive exercise.

Figure 8 shows one example frame towards the end of a two minute squat exercise of a virtual human character. On the left, the character is rendered with a standard subsurface scattering skin shader. On the right, the character show signs of exhaustion. We demonstrate her flushing, turning more red, starting to perspire, and deforming her chest with a deeper respiratory pattern (please see video). The right character appears more fatigued than the left character.

\subsection{Temporal Changes}

In the data analysis, changes are detected in the biosignal data correspond to changes in the motion of our captured subjects. Figure 12 shows a male subject performing jumping jacks. At the beginning of the motion he does not appear fatigued. He raises his arms high and cleanly performs the motion. As time goes on he is unable to raise his arms

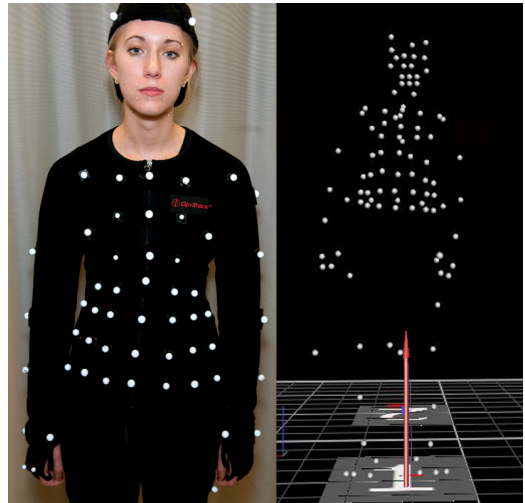

Figure 9: The left image shows the dense marker rig on a capture subject. The right image shows the markers reconstructed in Vicon's software.

up, and begins to appear more fatigued. A second example is shown in Figure 7. Here we plot the trajectory of the head, respiration, skin temperature, and skin conductance as a unathletic female subject performs squats. In the beginning of the motion, the subject breathes before she begins a squat. As the motion progresses, the breathing pattern becomes a bit erratic, the skin temperature falls, and she starts to dip down further in the squat, wobbling, and takes longer to get back up. This shows there is a clear correspondence between the biodata changes and changes in the skeletal motion. This correspondence is one of the major signs of fatigue.

\section{Conclusion and Future Work}

We have demonstrated a novel approach for animating exhaustion and fatigue effects by synchronized biosignal and motion capture data. The fatigue model produces virtual characters that appear more fatigued than simply playing 


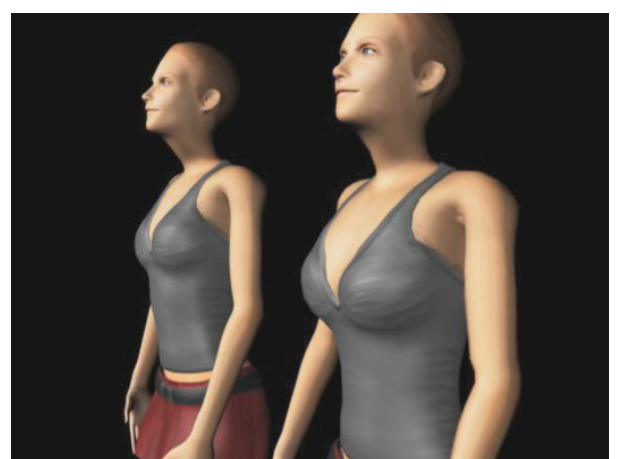

Figure 10: The left image shows the model's breathing deformation. The right image shows the dense set marker's deformation. We see that there is no visual difference between the two chests.

back motion capture data. We simulated an anatomically inspired human to generate these effects without having to do performance capture using an expensive dense marker set. The breathing, flushing, and perspiration effects enhanced the virtual character's appearance. We compared our model to simple sinusoidal breathing models, and dense marker set captures. Our method produced more realistic effects than the naïve models without a large quality loss from the dense marker set captures. Compared to tedious processing of dense marker sets, the fatigue model simulation offers a low overhead add-on to an existing animation pipeline.

The current model only focuses on certain fatigue effects. Other equally important aspects affecting human exercise motion such as muscle deformation, facial expressions, wrinkles, and eye movement are not being considered in this paper. All of these change over the course of a intensive exercise. We hope to layer some of these effects on the model in the future.

We plan to develop an even more robust model of the visual appearance. Some limitations of the fatigue effects lie in that we are not considering all the complex processes of the human body. Finding a blood oxygen sensor would greatly enhance how we could simulate oxygenation of the skin and body. Also, there are sensors that measure the internal body temperature of a subject, however they are extremely invasive to wear. We opted not to stick sensors inside the subject as they performed the exercise motions. However, internal temperature data would produce more accurate physiological effects.

In the future, we want to compare the data across exercise classes to see how they correlate with different changes in motion. The analysis of the biosignal channel user study data shows the results produced during the captured exercises are very repeatable. We plan to parameterize the deformations and fatigue effect and learn these parameters to layer the effects on motion without having to always cap- ture synchronized data. For example, people who are more athletic and fit tire differently than less fit individuals. The fatigue parameters will help us apply the proper exhaustion effects to characters of varying build and fitness.

\section{References}

[ACP02] Allen B., Curless B., Popović Z.: Articulated body deformation from range scan data. In ACM SIGGRAPH 2002 Papers (New York, NY, USA, 2002), SIGGRAPH '02, ACM, pp. 612-619.

[BHPS10] Bradley D., Heidrich W., Popa T., Sheffer A.: High resolution passive facial performance capture. In ACM SIGGRAPH 2010 papers (New York, NY, USA, 2010), SIGGRAPH '10, ACM, pp. 41:1-41:10.

[DCFN06] Deng Z., Chiang P.-Y., Fox P., Neumann U.: Animating blendshape faces by cross-mapping motion capture data. In Proceedings of the 2006 symposium on Interactive $3 D$ graphics and games (New York, NY, USA, 2006), I3D '06, ACM, pp. 43-48.

[Di109] DiLORENZO P. C.: Breathing, laughing, sneezing, coughing: model and control of an anatomically inspired, physicallybased human torso simulation. PhD thesis, University of California, Riverside, USA, 2009. AAI3350078.

[DZS08] Dilorenzo P. C., Zordan V. B., SANDERS B. L.: Laughing out loud: control for modeling anatomically inspired laughter using audio. In ACM SIGGRAPH Asia 2008 papers (New York, NY, USA, 2008), SIGGRAPH Asia '08, ACM, pp. 125:1-125:8.

[INN07] Igarashi T., Nishino K., NAYAR S. K.: The appearance of human skin: A survey. Found. Trends. Comput. Graph. Vis. 3 (January 2007), 1-95.

[JSB*10] Jimenez J., SCully T., Barbosa N., Donner C., Alvarez X., Vieira T., Matts P., Orvalho V., GutierREZ D., WEYRICH T.: A practical appearance model for dynamic facial color. In ACM SIGGRAPH Asia 2010 papers (New York, NY, USA, 2010), SIGGRAPH ASIA '10, ACM, pp. 141:1141:10.

[KHYS02] Kähler K., Haber J., Yamauchi H., Seidel H.-P.: Head shop: generating animated head models with anatomical structure. In Proceedings of the 2002 ACM SIGGRAPH/Eurographics symposium on Computer animation (New York, NY, USA, 2002), SCA '02, ACM, pp. 55-63.

[KMP97] Kaye J., Metaxas D. N., Primiano JR. F. P.: A 3d virtual environment for modeling mechanical cardiopulmonary interactions. In Proceedings of the First Joint Conference on Computer Vision, Virtual Reality and Robotics in Medicine and Medial Robotics and Computer-Assisted Surgery (London, UK, 1997), CVRMed-MRCAS '97, Springer-Verlag, pp. 389-398.

[KMT02] Kalra P., Magnenat-Thalmann N.: Modelling of vascular expressions in facial animation. In Proceedings of Computer Animation (2002), pp. 50-58.

[LM88] LAMB D., MurRy R.: Perspectives in Exercise Science and Sports Medicine. Benchmark Press, 1988.

[LT06] LEE S.-H., Terzopoulos D.: Heads up!: biomechanical modeling and neuromuscular control of the neck. In $A C M$ SIGGRAPH 2006 Papers (New York, NY, USA, 2006), SIGGRAPH '06, ACM, pp. 1188-1198.

[MAP*82] Mellander S., Andersson, P.O. A., L.E., Hellstrand P.: Neural beta-adrenergic dilatation of the facial vein in man: possible mechanism in emotional blushing. In Acta Physiologica Scandinavica (1982), pp. 393-399. 
(a) Subject 1
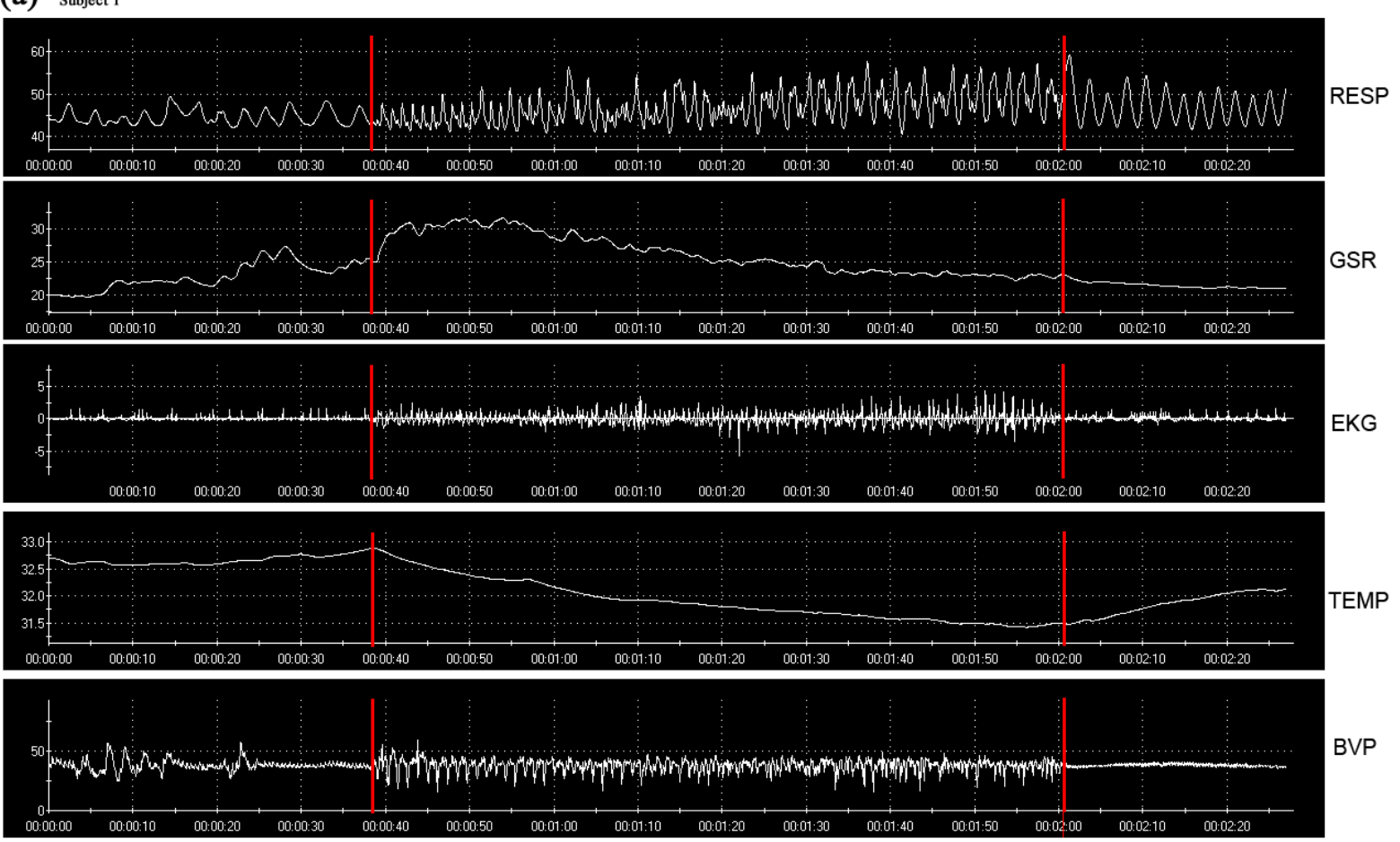

(b)

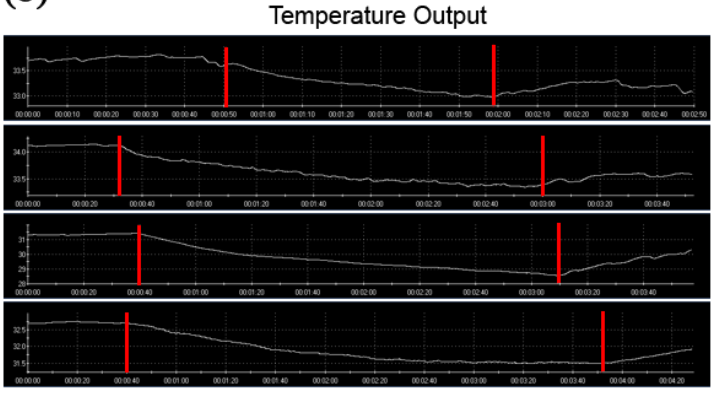

(c)

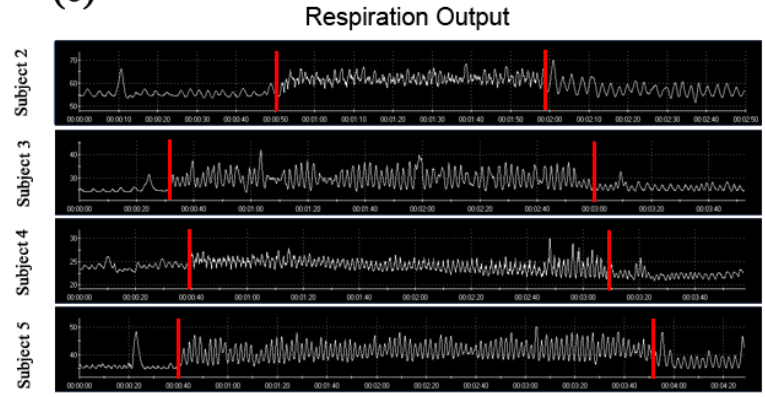

Figure 11: This graph shows the biodata capture of 5 different subjects from the user experiment. For each motion capture take the subject was asked to idle, perform an exercise (area between red lines on plots), then idle to cool down. There is a major difference in the biodata during an exercise. (a) shows Subject 1's (Male 180lbs athletic build): respiration, galvanic skin response, electrocardiography, skin temperature, and blood volume pulse for a jumping jack exercise, (b) and (c) show two male and two female subjects' biodata for squats, jumping jacks, and stair climbing exercises. (b) shows four subjects' skin temperature signal. All four subjects have similar thermal events: warm blood moving from the skin to the body core, and producing perspiration to cool down temperature during the exercise. Warm blood then returns to the skin immediately after the exercise. (c) shows four subjects' respiration signal. Again, all the subjects' breathing patterns become deeper and more vigorous during the exercise than when they idled. Towards the end of the exercise, as fatigue sets in the respiration signal shows the deeper breaths people take.

[Mar98] MARIEB E.: Human Anatomy and Physiology. Addision Wesley, 1998.

[MB91] Monheit G., BAdLer N. I.: A kinematic model of the human spine and torso. IEEE Comput. Graph. Appl. 11 (March 1991), 29-38.

[Met97] Metaxas D. N.: Physics-Based Deformable Models.
Kluwer Academic Publishers, 1997.

[MG09] MELo C. M., GRATCH J.: Expression of emotions using wrinkles, blushing, sweating and tears. In Proceedings of the 9th International Conference on Intelligent Virtual Agents (Berlin, Heidelberg, 2009), IVA '09, Springer-Verlag, pp. 188-200.

[Min93] Mines A.: Respiratory Physiology. Raven Press, 1993.

(c) The Eurographics Association 2011. 


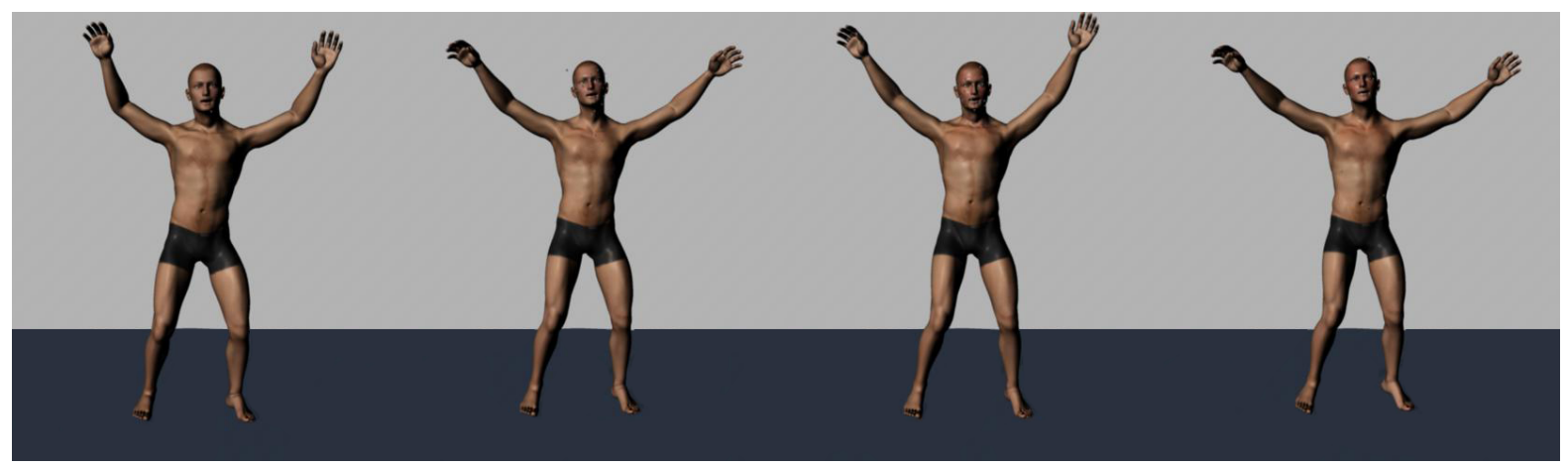

Figure 12: This figure shows a male subject performing jumping jacks. In the left frame, he appears energetic. In successive frames to the right he starts to flush, sweat, and pant more. Also, at the start of the motion (far left) he raises his arms high, towards the end (far right) he appears more exhausted and cannot lift his arms as high. This shows the biodata and the motion change together.

[MTCS08] Magnenat-Thalmann N., Charbonnier C., SCHMID J.: Multimedia application to the simulation of human musculoskeletal system: A visual lower limb model from multimodal captured data. In Multimedia Signal Processing, 2008 IEEE 10th Workshop on (oct. 2008), pp. 520-525.

[PB81] Platt S. M., BADlER N. I.: Animating facial expressions. In Proceedings of the 8th annual conference on Computer graphics and interactive techniques (New York, NY, USA, 1981), SIGGRAPH '81, ACM, pp. 245-252.

[PBP97] Promayon E., Baconnier P., Puech C.: Physicallybased model for simulating the human trunk respiration movements. In Proceedings of the First Joint Conference on Computer Vision, Virtual Reality and Robotics in Medicine and Medial Robotics and Computer-Assisted Surgery (London, UK, 1997), CVRMed-MRCAS '97, Springer-Verlag, pp. 379-388.

[PH06] PARK S. I., Hodgins J. K.: Capturing and animating skin deformation in human motion. ACM Trans. Graph. 25 (July 2006), 881-889

[PH08] PARK S. I., Hodgins J. K.: Data-driven modeling of skin and muscle deformation. In ACM SIGGRAPH 2008 papers (New York, NY, USA, 2008), SIGGRAPH '08, ACM, pp. 96:196:6.

[PT57] PRICE Thomas I. K.: Relationship between sweat gland activity and electrical resistance of the skin. In Journal of Applied Physiology (1957), pp. 578-586.

[Sag06] SAGAR1 M.: Facial performance capture and expressive translation for king kong. In ACM SIGGRAPH 2006 Courses (New York, NY, USA, 2006), SIGGRAPH '06, ACM.

[SCCH09] Shiratori T., COley B., Cham R., Hodgins J. K.: Simulating balance recovery responses to trips based on biomechanical principles. In Proceedings of the 2009 ACM SIGGRAPH/Eurographics Symposium on Computer Animation (New York, NY, USA, 2009), SCA '09, ACM, pp. 37-46.

[SDZB08] SANDERS B., Dilorenzo P., Zordan V., BAKAL D.: Toward anatomical simulation for breath retraining in mind/body medicine. In Workshop on 3D Physiological Human (2008).

[SMP03] Sand P., McMillan L., Popović J.: Continuous capture of skin deformation. In ACM SIGGRAPH 2003 Papers (New York, NY, USA, 2003), SIGGRAPH '03, ACM, pp. 578586.
[SNF05] Sifakis E., Neverov I., FedKIW R.: Automatic determination of facial muscle activations from sparse motion capture marker data. In ACM SIGGRAPH 2005 Papers (New York, NY, USA, 2005), SIGGRAPH '05, ACM, pp. 417-425.

[TLX10] Tengyu Z., Lifeng L., Xiaojun S.: Design and application of gait analysis system for prosthesis wearers. In Proceedings of the 4th International Convention on Rehabilitation Engineering \& Assistive Technology (Kaki Bukit TechPark II, Singapore, 2010), iCREATe '10, Singapore Therapeutic, Assistive \& Rehabilitative Technologies (START) Centre, pp. 53:1$53: 3$.

[vic11] Vicon motion systems, May 2011.

[Wat87] WATERS K.: A muscle model for animation threedimensional facial expression. In Proceedings of the 14th annual conference on Computer graphics and interactive techniques (New York, NY, USA, 1987), SIGGRAPH '87, ACM, pp. 1724.

[Wes04] West J.: Respiratory Physiology: the essentials. Lippincott Williams \& Wilkins, 2004.

[WMP*06] Weyrich T., Matusik W., Pfister H., Bickel B., Donner C., Tu C., McAndless J., Lee J., NGan A., Jensen H. W., Gross M.: Analysis of human faces using a measurement-based skin reflectance model. In ACM SIGGRAPH 2006 Papers (New York, NY, USA, 2006), SIGGRAPH '06, ACM, pp. 1013-1024.

[YW07] YAMADA T., WATANABE T.: Virtual facial image synthesis with facial color enhancement and expression under emotional change of anger. In 16th IEEE International Conference on Robot \& Human Interactive Communication, pp. 49-54.

[ZCCD06] Zordan V. B., Celly B., Chiu B., Dilorenzo P. C.: Breathe easy: model and control of human respiration for computer animation. Graphical Models 68 (March 2006), 113132 . 